\title{
Compartment Syndrome Following a Total Knee Replacement
}

\author{
Amit Chauhan, MBBS, MS, MRCS ${ }^{1}$ Shoaib Khan, MBBS, MRCS ${ }^{1}$ Bimal Singh, MBBS, MS, FICS ${ }^{1}$ \\ Shanmuga Maheswaran, FRCS ${ }^{1}$ Nicholas Cooke, FRCS ${ }^{1}$ Siu Tang, FRCS ${ }^{1}$
}

${ }^{1}$ Department of Orthopedics, University Hospital of North Tees, Stockton on Tees, United Kingdom

J Knee Surg Rep 2015;1:64-68.
Address for correspondence Amit Chauhan, MBBS, MS, MRCS, Department of Orthopedics, University Hospital of North Tees, Hardwick Road Stockton on Tees, Stockton on Tees TS19 8PE, United Kingdom (e-mail: amitcoolest4@gmail.com).
Abstract
Keywords
- knee
- arthroplasty
- fasciotomy
- compartment syndrome

Compartment syndrome is a well-known entity. It has been seen more commonly after trauma and crush injuries. It is quite rare after a total knee arthroplasty. Various factors which can implicate this rare sequence have been highlighted. We report here a case of compartment syndrome of calf after an elective total knee arthroplasty, along with a review of literature. Patient was managed with urgent decompression with full recovery.
Compartment syndrome is an orthopedic emergency. A high index of suspicion, timely diagnosis, and early decompression are of prime importance. Several factors can cause this unfortunate incident leading to irreversible injury to muscle and nerves. It is quite rare after a total knee arthroplasty (TKA). Further, it becomes extremely difficult to timely diagnose compartment syndrome after such a procedure. Factors which can delay its diagnosis after a knee arthroplasty have been discussed. We report here a case of compartment syndrome of calf after a TKA, with review of relevant literature.

\section{Case Report}

A 67-year-old man was admitted to elective orthopedic unit for a TKA for osteoarthritis ( - Fig. 1A, B) of left knee. Patient was having arthritic symptoms of pain for the last 10 years affecting his daily routine activities. Past history included hiatus hernia, high cholesterol, a previous left partial medial meniscectomy, and a right total knee replacement with good recovery without any perioperative or postoperative complications. Patient did not have any coagulation or bleeding disorder and was not on any anticoagulant prior to surgery. His body mass index was 28.13. Drug history included lansoprazole, paracetamol, and statins. After complete preassessment, this gentleman was operated for this elective procedure under spinal anesthesia. Regional blocks or epidural anesthesia was not used. Tourniquet pressure and time were $300 \mathrm{~mm} \mathrm{Hg}$ and 89 minutes, respectively. A cruciatesacrificing cemented total knee replacement with patellar resurfacing was done with an uneventful immediate perioperative period. Around $300 \mathrm{~mL}$ of irrigation was used during the procedure with judicious use of monopolar diathermy. A low vacuum suction drain was used and anticoagulation, in the form of low molecular weight heparin (enoxaparin 3,500 IU s.c. once every evening), was started at night. Postoperatively, patient had OxyContin $20 \mathrm{mg}$ for pain relief on the first day which was taken off the following day. Both active and passive physiotherapy were started the same day, and patient was mobilizing well with the help of Zimmer frame, with 70 degrees flexion at 48 hours of surgery. On day 3, patient developed severe pain in left knee and leg region. On examination, skin staples were dry without any local soakage. Left calf was swollen, firm, and tender. Knee flexion was seen to be limited to only 20 degrees with restricted ipsilateral ankle movements. Peripheral pulses were normal in rate, rhythm, and volume. An urgent Doppler scan excluded deep venous thrombosis. Analgesic medication dosages were increased to relieve the symptoms of pain, and the routine physiotherapy session was withheld. Another examination done half an hour later revealed severe pain on passive stretching. Peripheral pulses were still present as before. A clinical diagnosis of received

November 26, 2014

accepted after revision

June 25, 2015

published online

September 21, 2015
DOI http://dx.doi.org/

10.1055/s-0035-1564709. ISSN 2326-2729.
Copyright (c) 2015 by Thieme Medical

Publishers, Inc., 333 Seventh Avenue, New York, NY 10001, USA. Tel: +1(212) 584-4662.
License terms

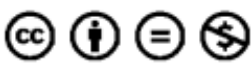




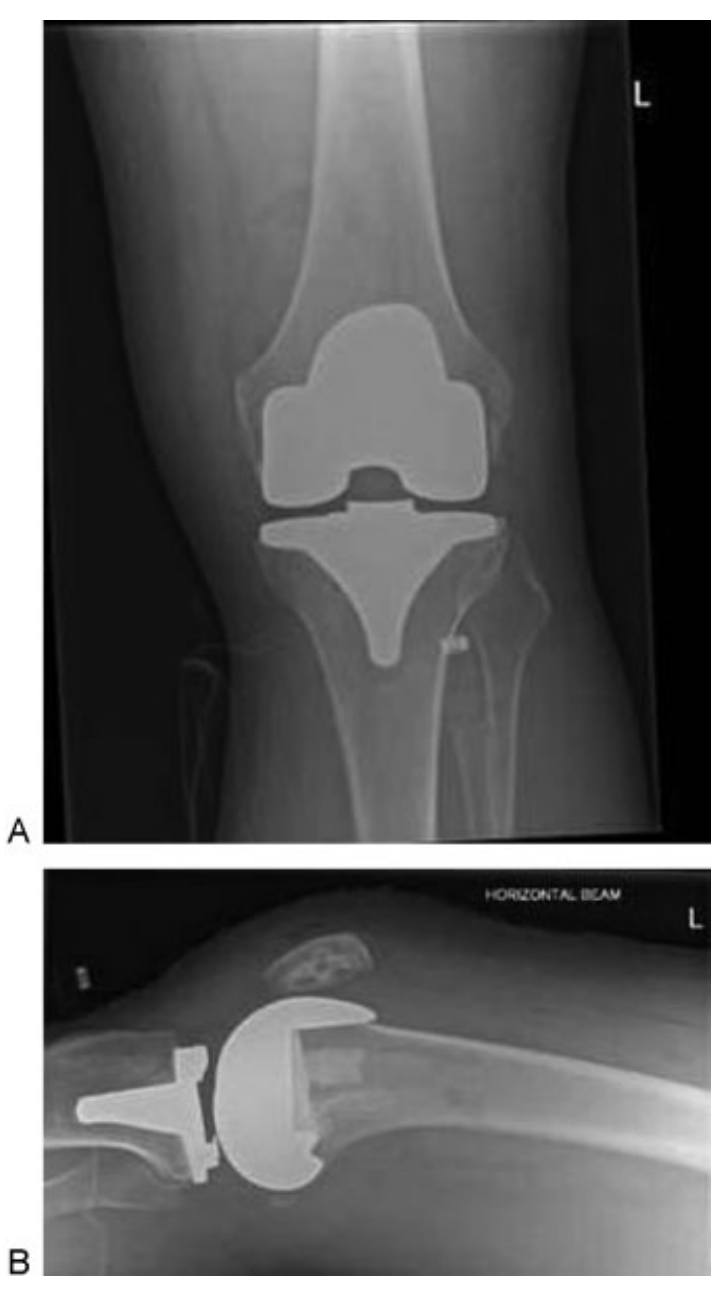

Fig. 1 (A and B) Anteroposterior and lateral view showing cemented total knee prosthesis in immediate postoperative period. compartment syndrome of calf led to an urgent fasciotomy using a two-incision technique with decompression of all four compartments. Compartment pressures were not measured for this urgent condition. Intraoperatively, muscle bulging out was prominent without any wound hematoma or vascular injury. The incised wounds were kept open and regularly dressed for two consecutive days with assessment of the wound and muscle color, consistency, and contraction. Closure was performed 3 days after fasciotomy ( - Fig. 2A-C). An uneventful recovery was seen without any neurologic deficit with range of motion from 10 degrees of extension to 95 degrees of knee flexion at 3-month follow-up.

\section{Discussion}

Compartment syndrome is a result of increased pressure in a closed fascial space compromising the circulation to the nerves and muscles within the involved compartment. ${ }^{1}$ It can be due to either increased external pressure, or increase in content volume of compartment, or a decrease in volume of affected compartment. Any of these factor can compromise local tissue perfusion. ${ }^{2}$ Early signs and symptoms consist of pain disproportionate to the injury, which is exacerbated by passive stretching of the involved muscles, swelling, and coldness. ${ }^{3}$ Pulselessness may or may not manifest and presence of peripheral pulse cannot rule out compartment syndrome. ${ }^{4}$

Compartment syndrome is an orthopedic emergency where clinical suspicion is of paramount importance, thus allowing early surgical treatment. Irreversible ischemic necrosis of muscles and nerves can cause significant morbidity in cases with missed or late diagnosis and late decompression. Neglected cases can have severe systemic complications such as myoglobinuria, renal failure, and even death. ${ }^{5}$ Compartment pressure measurement is helpful in unconscious
A

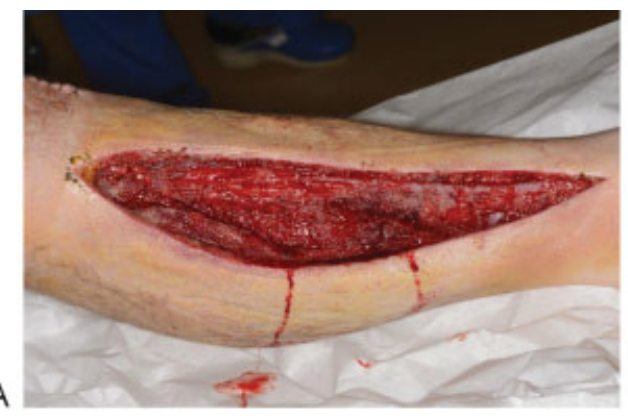

B

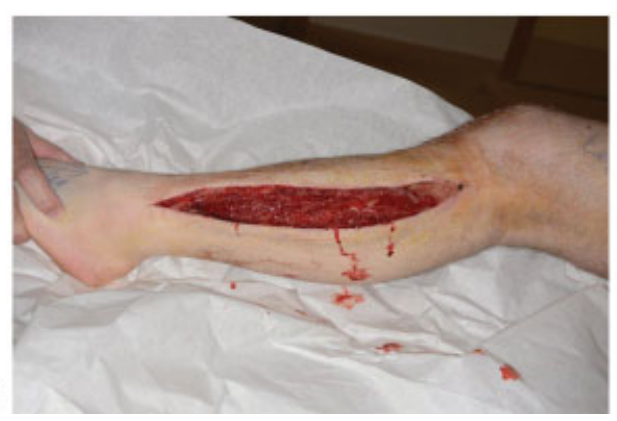

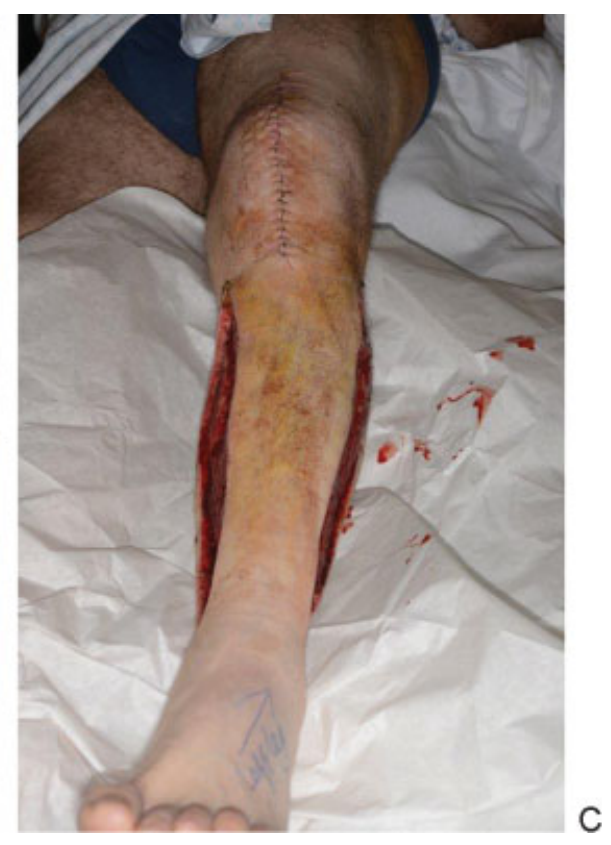

Fig. 2 (A, B, and C) Fasciotomy wound on day of closure. 


\begin{tabular}{|c|c|c|c|c|c|c|c|c|c|c|c|c|c|}
\hline 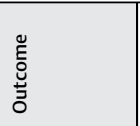 & 曲 & $\dddot{x}$ & 曲 & 窝 & 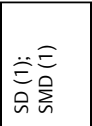 & 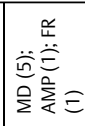 & 号 & 똔 & 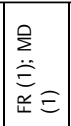 & in & 똔 & 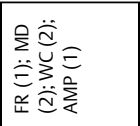 & 曲 \\
\hline 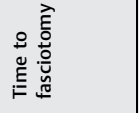 & $t$ & $\frac{\pi}{z}$ & $\frac{s}{z}$ & 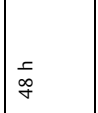 & $\mid \frac{f}{f}$ & 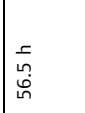 & 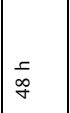 & $\underset{\sim}{\tilde{N}}$ & $\mid$\begin{tabular}{l}
\multicolumn{5}{c}{} \\
so
\end{tabular} & $\underset{f}{\tilde{N}}$ & $\frac{s}{z}$ & 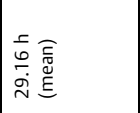 & 总 \\
\hline $\begin{array}{l}\frac{2}{0} \\
\frac{0}{2} \\
\sum_{\bar{z}}^{2}\end{array}$ & ż & z & $\frac{x}{z}$ & $\frac{\Sigma}{z}$ & 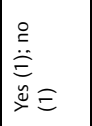 & 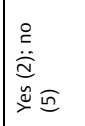 & 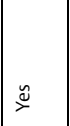 & $\stackrel{\check{\Xi}}{\searrow}$ & 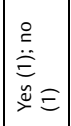 & $\frac{\pi}{z}$ & z & & z \\
\hline 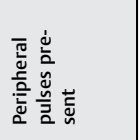 & $\stackrel{\check{\nu}}{\searrow}$ & $\stackrel{y}{x}$ & $\frac{x}{z}$ & $\stackrel{\check{\nu}}{\varnothing}$ & 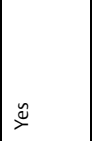 & 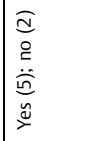 & i & $\stackrel{\circ}{2}$ & $\frac{s}{z}$ & $\frac{s}{z}$ & 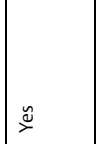 & $\frac{\sqrt{n}}{2}$ & $\cong$ \\
\hline 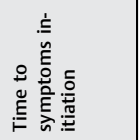 & 采 & 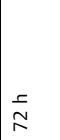 & $\frac{\pi}{z}$ & 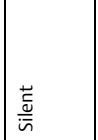 & 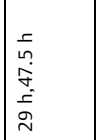 & $\frac{x}{z}$ & 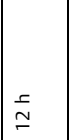 & $\stackrel{\mathfrak{I}}{\stackrel{I}{I}}$ & 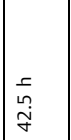 & $\underset{\sim}{\stackrel{f}{N}}$ & $\frac{s}{z}$ & 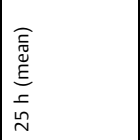 & 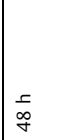 \\
\hline 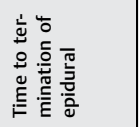 & $\frac{s}{z}$ & $\frac{x}{z}$ & $\frac{\pi}{z}$ & $\mid$\begin{tabular}{c}
\multicolumn{1}{c}{} \\
$\stackrel{\infty}{\infty}$
\end{tabular} & \begin{tabular}{|l}
$\tilde{r}$ \\
$\tilde{m}$ \\
$\dot{\sim}$ \\
$\tilde{N}$
\end{tabular} & 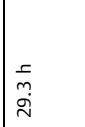 & $\frac{\pi}{z}$ & $\underset{\sim}{\stackrel{\sim}{\sim}}$ & 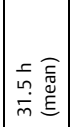 & 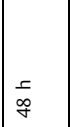 & $\frac{s}{z}$ & $\frac{\pi}{2}$ & $\frac{s}{z}$ \\
\hline 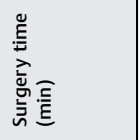 & $\frac{\pi}{z}$ & $\frac{\pi}{z}$ & $\frac{\pi}{z}$ & 号 & 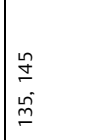 & 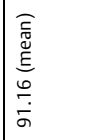 & ட & $\stackrel{i}{m}$ & 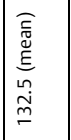 & $\frac{\pi}{z}$ & $\theta$ & 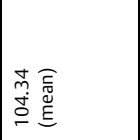 & 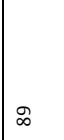 \\
\hline 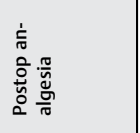 & 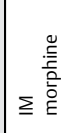 & 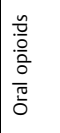 & $\frac{\pi}{z}$ & u & u & 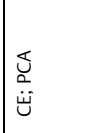 & u & u & u & u & 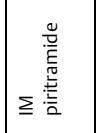 & 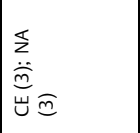 & 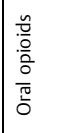 \\
\hline 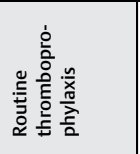 & $\stackrel{\check{\Xi}}{~}$ & 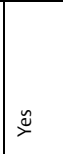 & $\frac{x}{z}$ & $\frac{\pi}{z}$ & $\frac{\pi}{z}$ & 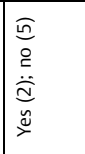 & $\stackrel{\searrow}{\rightleftharpoons}$ & $\frac{\pi}{z}$ & $\frac{\pi}{z}$ & $\frac{\pi}{z}$ & $\stackrel{\check{x}}{\searrow}$ & 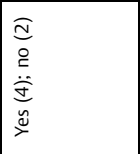 & $\stackrel{\check{\beth}}{\check{\nu}}$ \\
\hline 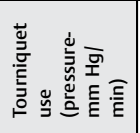 & 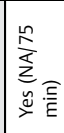 & $\frac{x}{z}$ & $\frac{\pi}{z}$ & 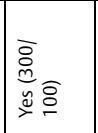 & 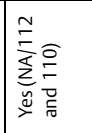 & 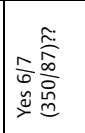 & 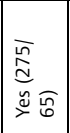 & 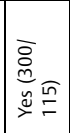 & 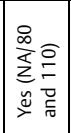 & 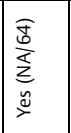 & 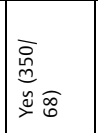 & 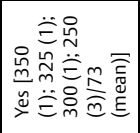 & 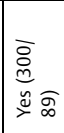 \\
\hline 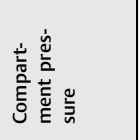 & $\stackrel{m}{m}$ & 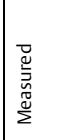 & $\frac{x}{z}$ & 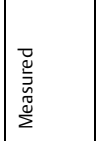 & $\frac{s}{z}$ & 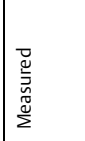 & $\frac{s}{z}$ & $\frac{\pi}{z}$ & $\frac{\pi}{z}$ & $\frac{s}{z}$ & 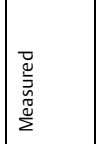 & 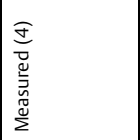 & $\frac{s}{z}$ \\
\hline 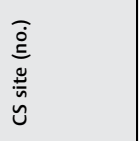 & \begin{tabular}{|c} 
总 \\
官
\end{tabular} & 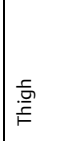 & 㱒 & 㱒 & 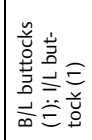 & 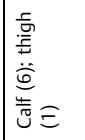 & 陟 & 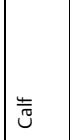 & 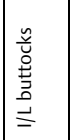 & $\frac{4}{\mathbb{J}}$ & 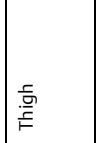 & $\frac{\sqrt{\pi}}{\pi}$ & $\frac{4}{\pi}$ \\
\hline 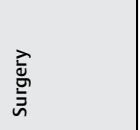 & 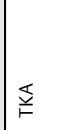 & $\stackrel{\mathbb{F}}{\stackrel{\Xi}{F}}$ & $\underline{F}$ & 采 & $\underset{\xi}{\stackrel{\Xi}{F}}$ & 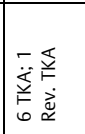 & $\underset{F}{\stackrel{\Xi}{F}}$ & 竝 & 䋨 & 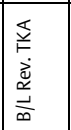 & 站 & 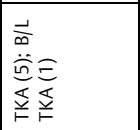 & 㠩 \\
\hline 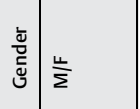 & $\Sigma$ & $\Sigma$ & $\sqcup$ & 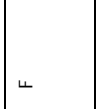 & $\sum_{N}^{\Sigma}$ & \begin{tabular}{|l} 
\\
$\square$ \\
$\dot{\Sigma}$ \\
$m$
\end{tabular} & 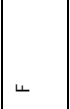 & $\sqcup$ & 峞 & $\frac{\pi}{2}$ & $\Sigma$ & $\mid$ & $\Sigma$ \\
\hline 过 & 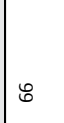 & 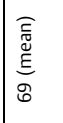 & 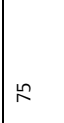 & 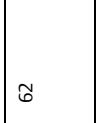 & in & 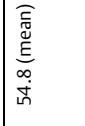 & F & F & 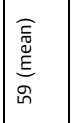 & $\frac{\pi}{z}$ & $\widetilde{\sigma}$ & 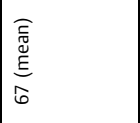 & to \\
\hline 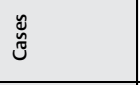 & - & m & - & - & $\sim$ & n & - & - & $\sim$ & - & - & 0 & - \\
\hline 产 & 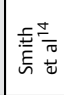 & 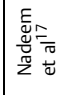 & 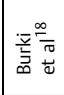 & 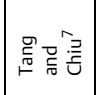 & 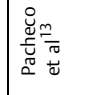 & 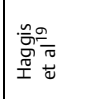 & 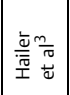 & 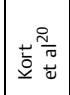 & 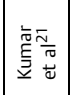 & 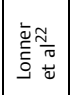 & 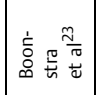 & 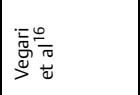 & 言竞 \\
\hline
\end{tabular}


patients and in rare, clinically inconclusive scenarios and where regional anesthesia is used. Although critical pressure at which decompression is required remains controversial, ${ }^{6}$ this measurement results in earlier decompression and less dysfunction. ${ }^{4}$ Fasciotomy is recommended when compartment pressure exceeds 30 to $35 \mathrm{~mm} \mathrm{Hg}{ }^{1}$

A lot of factors are relevant in delaying early diagnosis of compartment syndrome after a TKA. Epidural anesthesia contributes by masking the symptoms. ${ }^{7}$ Neurovascular injuries after TKA such as peroneal nerve palsy and vascular injury can delay the diagnosis. ${ }^{8}$ Tourniquet use can cause compartment syndrome. An absolute upper limit of safety of 3 hours and pressure for the thigh to be double the systolic pressure in arm have been suggested. ${ }^{9}$ Extensive soft tissue dissection in presence of previous scarring can cause bleeding. Further, anticoagulant prophylaxis, routinely given to prevent venous thromboembolism, can have serious hemorrhagic risks. ${ }^{10}$ In addition, symptoms such as swelling and calf pain may also suggest deep venous thrombosis, and further increase the diagnostic dilemma of the attending clinician. Role of calf compression devices remains unclear. They have ironically been both implicated in causation ${ }^{11}$ and known to decrease compartment pressures by improving venous return. ${ }^{12}$ Following TKA, physiotherapy in the form of early continuous passive motion with flexion to 30 degrees has been shown to increase compartment pressures up to $35 \mathrm{~mm}$ $\mathrm{Hg}$ in thigh or gluteal compartment. ${ }^{13,14}$ Obesity, previous surgery on same joint, comorbid vascular disease, prolonged surgery, and hypotensive anesthesia have also been implicated. Lastly, knowledge and clinical acumen of supporting staff involved in perioperative care cannot be underestimated. ${ }^{15}$ These variables, as found in literature search on this rare event, have been tabulated $3,7,13,14,16-23$ (- Table 1).

Moreover, it has been reported that if decompression is performed within 8 hours of initiation of symptoms, a successful defense for an orthopedic surgeon is possible. ${ }^{24}$ Legal issues are increasingly being reported ${ }^{16}$ and some do result in an avoidable compensation. The cause of compartment syndrome in our case, even after careful evaluation of above-mentioned factors, still eludes us.

\section{Conclusion}

To conclude, compartment syndrome following a TKA is extremely rare. It requires awareness among staff, a watchful clinician, and a very low threshold for decompression to minimize morbidity following this unfortunate event. Various factors as outlined in this report should be able to help in clinical judgment when suspecting compartment syndrome following a TKA.

\section{References}

1 Mubarak SJ, Owen CA, Hargens AR, Garetto LP, Akeson WH. Acute compartment syndromes: diagnosis and treatment with the aid of the wick catheter. J Bone Joint Surg Am 1978;60(8):1091-1095
2 Pelligrini VD, Reid JS, Evarts CM. Complications. In: Rockwood CA, Green DP, Bucholz RW, Heckman JD, eds. Fractures in Adults. 3rd ed. Philadelphia, PA: Lippincott-Raven; 1996:487

3 Hailer NP, Adalberth G, Nilsson OS. Compartment syndrome of the calf following total knee arthroplasty-a case report of a highly unusual complication. Acta Orthop 2007;78(2):293-295

4 McQueen M. Acute compartment syndrome. Acta Chir Belg 1998; 98(4):166-170

5 Ebenezer S, Dust W. Missed acute isolated peroneal compartment syndrome. CJEM 2002;4(5):355-358

6 Köstler W, Strohm PC, Südkamp NP. Acute compartment syndrome of the limb. Injury 2004;35(12):1221-1227

7 Tang WM, Chiu KY. Silent compartment syndrome complicating total knee arthroplasty: continuous epidural anesthesia masked the pain. J Arthroplasty 2000;15(2):241-243

8 Ayers DC, Dennis DA, Johanson NA, Pellegrini VD. Common complications of total knee arthroplasty. J Bone Joint Surg Am 1997;79-A:278-311

9 Klenerman L, Hulands GH. Tourniquet pressures for the lower limb. J Bone Joint Surg Br 1979;61B:124-128

10 Hynson JM. Role of heparin in compartment syndrome. J Bone Joint Surg Am 2000;82(5):752-753

11 Lachmann EA, Rook JL, Tunkel R, Nagler W. Complications associated with intermittent pneumatic compression. Arch Phys Med Rehabil 1992;73(5):482-485

12 Pfeffer SD, Halliwill JR, Warner MA. Effects of lithotomy position and external compression on lower leg muscle compartment pressure. Anesthesiology 2001;95(3):632-636

13 Pacheco RJ, Buckley S, Oxborrow NJ, Weeber AC, Allerton K. Gluteal compartment syndrome after total knee arthroplasty with epidural postoperative analgesia. J Bone Joint Surg Br 2001;83(5): 739-740

14 Smith PN, Rampersaud R, Rorabeck CH. Incipient compartment syndrome of the thigh following total knee arthroplasty. J Arthroplasty 1997;12(7):835-838

15 Lasanianos NG, Kanakaris NK, Roberts CS, Giannoudis PV. Compartment syndrome following lower limb arthroplasty: a review. Open Orthop J 2011;5:181-192

16 Vegari DN, Rangavajjula AV, Diiorio TM, Parvizi J. Fasciotomy following total knee arthroplasty: beware of terrible outcome. J Arthroplasty 2014;29(2):355-359

17 Nadeem RD, Clift BA, Martindale JP, Hadden WA, Ritchie IK. Acute compartment syndrome of the thigh after joint replacement with anticoagulation. J Bone Joint Surg Br 1998;80(5):866-868

18 Burki H, von Knoch M, Heiss C, Drobny T, Munzinger U. Lateral approach with osteotomy of the tibial tubercle in primary total knee arthroplasty. Clin Orthop Relat Res 1999;(362):156-161

19 Haggis P, Yates P, Blakeway C, et al. Compartment syndrome following total knee arthroplasty: a report of seven cases. J Bone Joint Surg Br 2006;88(3):331-334

20 Kort NP, van Raay JJ, van Horn JR. Compartment syndrome and popliteal vascular injury complicating unicompartmental knee arthroplasty. J Arthroplasty 2007;22(3):472-476

21 Kumar V, Saeed K, Panagopoulos A, Parker PJ. Gluteal compartment syndrome following joint arthroplasty under epidural anaesthesia: a report of 4 cases. J Orthop Surg (Hong Kong) 2007; 15(1):113-117

22 Lonner JH, Jasko JG, Bezwada HP, Booth RE Jr. Morbidity of sequential bilateral revision TKA performed under a single anesthetic. Clin Orthop Relat Res 2007;464(464):151-156

23 Boonstra RH, Haverkamp D, Campo MM, van der Vis HM. Acute compartment syndrome of the thigh following total knee arthroplasty. Knee 2012;19(2):151-153

24 Bhattacharyya T, Vrahas MS. The medical-legal aspects of compartment syndrome. J Bone Joint Surg Am 2004;86-A(4): 864-868 